\title{
The Impact of Carbapenem Resistance on Mortality in Patients With Klebsiella Pneumoniae Bloodstream Infection: An Individual Patient Data Meta-Analysis of 1952 Patients
}

\author{
Alberto E. Maraolo - Silvia Corcione - Adriano Grossi · Alessio Signori • Cristiano Alicino $\cdot$ Khetam Hussein • \\ Enrico M. Trecarichi · Pierluigi Viale · Jean-François Timsit • Balaji Veeraraghavan · Maria V. Villegas • \\ Galia Rahav • George L. Daikos · Konstantinos Z. Vardakas • Emmanuel Roilides • Anne-Catrin Uhlemann • \\ Abdul K. Ghafur · Simone Mornese Pinna · Matteo Bassetti · Philipp P. Kohler · Daniele R. Giacobbe
}

Received: October 30, 2020 / Accepted: January 23, 2021 / Published online: February 14, 2021

(C) The Author(s) 2021

\section{ABSTRACT}

Introduction: Available evidence from observational studies and meta-analyses has highlighted an increased mortality in patients with carbapenem-resistant Klebsiella pneumoniae

The present project is endorsed by SITA GIOVANI (Young Investigators Group of the Italian Society of Anti-Infective Therapy)

Supplementary Information The online version contains supplementary material available at https:// doi.org/10.1007/s40121-021-00408-8.

A. E. Maraolo $(\bowtie)$

First Division of Infectious Diseases, Cotugno

Hospital, AORN Dei Colli, Naples, Italy

e-mail: albertomaraolo@mail.com

S. Corcione $\cdot$ S. Mornese Pinna

Department of Medical Sciences, Infectious

Diseases, University of Turin, Turin, Italy

A. Grossi

Section of Hygiene, University Department of Life Sciences and Public Health, University Cattolica del Sacro Cuore, Rome, Italy

A. Signori - C. Alicino - M. Bassetti - D. R. Giacobbe Department of Health Sciences (DISSAL), University of Genoa, Genoa, Italy

\section{Alicino}

Medical Direction, Santa Corona Hospital, ASL 2 Regional Health System of Liguria, Pietra Ligure, Italy
(CRKP) bloodstream infections (BSI) compared with their carbapenem-susceptible (CSKP) counterparts, but the exact reasons for this outcome difference are still to be determined.

Methods: We updated the search of a previous meta-analysis through four databases up to April 2018. A two-stage individual-patient data (IPD) meta-analysis was conducted, building an adjusting model to account for age, comorbidities and activity of empirical and targeted antimicrobial therapy. The protocol was registered on PROSPERO (identifier: CRD42018104256).

\section{K. Hussein}

Infectious Diseases Unit, Rambam Health Care Campus, Haifa, Israel

E. M. Trecarichi

Department of Medical and Surgical Sciences, Infectious and Tropical Diseases Unit, "Magna Graecia" University, Catanzaro, Italy

P. Viale

Department of Medical and Surgical Sciences, Clinics of Infectious Diseases, S. Orsola-Malpighi Hospital, "Alma Mater Studiorum" University of Bologna, Bologna, Italy

\section{J.-F. Timsit}

AP-HP, Bichat Claude Bernard Hospital, Medical and Infectious Diseases ICU (MI2), Paris, France

B. Veeraraghavan

Department of Clinical Microbiology, Christian

Medical College, Vellore, Tamil Nadu, India 
Results: IPD data were obtained from 14 out of 28 eligible observational studies. A total of 1952 patients were investigated: 1093 in the CRKP group and 859 in the CSKP group. Patients with CRKP-BSI had a twofold risk of death compared with CSKP-infected patients [adjusted odds ratio (aOR) 2.17; 95\% confidence interval (CI) $\left.1.56-3.04 ; I^{2}=44.1 \%\right]$. Mortality was higher in patients with CRKP BSI, in both the subgroup of absent/inactive (aOR 1.75; 95\% CI 1.24-2.47; $I^{2}=0$ ) and of active initial therapy (aOR 2.66; 95\% CI 1.70-4.16; $I^{2}=16 \%$ ) as well as in case of active targeted therapy (aOR 2.21; 95\% CI $\left.1.36-3.59 ; I^{2}=58 \%\right)$.

Conclusion: Resistance to carbapenem is associated with worse outcome in patients with BSI by Klebsiella pneumoniae even adjusting for comorbidities and treatment appropriateness according to in vitro activity of empirical and targeted therapy. This applies to a scenario dominated by colistin-based therapies for CRKP. Further studies are needed to compare the mortality difference between CRKP and CSKP cases in the light of new anti-CRKP antimicrobials.

Keywords: Active therapy; Carbapenemresistant Klebsiella pneumoniae; Individual patient data meta-analysis; Monotherapy

M. V. Villegas

Grupo de Resistencia Antimicrobiana Y

Epidemiología Hospitalaria, Universidad El Bosque, Bogotá, Colombia

G. Rahav

Infectious Diseases Unit, The Chaim Sheba Medical Center, Tel Hashomer, Ramat Gan, Israel

G. L. Daikos

First Department of Medicine, Laiko General

Hospital, National and Kapodistrian University of

Athens, Athens, Greece

K. Z. Vardakas

Alfa Institute of Biomedical Sciences (AIBS), Athens, Greece

K. Z. Vardakas

Department of Medicine, Henry Dunant Hospital

Center, Athens, Greece

\section{Key Summary Points}

\section{Why carry out this study?}

Mortality is higher in patients with carbapenem-resistant Klebsiella pneumoniae (CRKP) bloodstream infections (BSI) than in patient with carbapenem-susceptible K. pneumonia (CSKP) BSI.

This study tried to provide an insight into the reasons underlying this mortality difference exploiting the advantages of an individual patient data (IPD) metaanalysis.

\section{What was learned from the study?}

BSI by CRKP is associated with a worse outcome compared with CSKP BSI even adjusting for comorbidities and treatment appropriateness according to in vitro activity of empirical and targeted therapy.

This study confirmed the increased mortality associated with resistance to carbapenems in KP BSI, at least in a scenario of colistin-based therapy.

Further studies are needed to address the role of virulence and the impact of novel drugs anti-CRKP.

\section{E. Roilides}

Infectious Disease Unit and Third Department of Pediatrics, Faculty of Medicine, Aristotle University

School of Health Sciences, Hippokration Hospital, Thessaloniki, Greece

\section{A.-C. Uhlemann}

Department of Medicine, Division of Infectious Diseases, Columbia University Irving Medical Center, New York, NY, USA

\section{A. K. Ghafur}

Apollo Cancer Institute, Anna Salai, Chennai, India

M. Bassetti · D. R. Giacobbe

Ospedale Policlinico San Martino, IRCCS Per

L'Oncologia, L. go R. Benzi 10, Genoa, Italy 


\section{DIGITAL FEATURES}

This article is published with digital features, including a summary slide, to facilitate understanding of the article. To view digital features for this article go to https://doi.org/10.6084/ m9.figshare.13622747.

\section{INTRODUCTION}

Carbapenem-resistant K. pneumoniae (CRKP) bloodstream infections (BSI) are a major public health issue worldwide, being responsible for multiple nosocomial outbreaks burdened by high morbidity and mortality [1-3]. In 2017, the World Health Organization included CRKP among the critical antibiotic-resistant bacterial pathogens for which novel effective drugs are urgently needed [4].

In line with these concerns, many observational studies and meta-analyses have highlighted an increased mortality in patients with CRKP BSI than in patients with carbapenemsusceptible K. pneumoniae (CSKP) BSI [5-7]. However, what still remain partly unclear are the true reasons underlying this difference in mortality. Indeed, while an increased risk of inappropriate (defined as inactive in vitro) empiric therapy against CRKP compared with against CSKP very likely contributes as an important explanatory factor, there is no definite proof that it fully explains the observed difference.

In the present study, we exploited the advantages of an individual patient data (IPD) meta-analysis [8] in the attempt to increase our understanding of the reasons underlying this mortality difference.

\section{METHODS}

The protocol of the present study was registered in the PROSPERO database in August 2018 (registration number: CRD42018104256). We

P. P. Kohler

Division of Infectious Diseases and Hospital

Epidemiology, Cantonal Hospital St. Gallen, St.

Gallen, Switzerland carried out the systematic review and IPD metaanalysis according to the PRISMA-IPD (Preferred Reporting Items for Systematic Reviews and Meta-Analyses: Individual Participant Data) guidelines [9]. The PRISMA-IPD checklist is provided in a dedicated appendix. The current work is an expansion of a previous (non-IPD) meta-analysis on the same topic [7].

\section{Eligibility Criteria}

A complete and detailed list of inclusion and exclusion criteria is available in Table 1. Overall, we retained the inclusion and exclusion criteria set by the previous meta-analysis [7]; additionally, we introduced additional criteria based on the availability of specific patient-level data. Inclusion criteria relied on the PECO strategy: studies whose subjects were adult hospitalized individuals ( $\mathrm{P}=$ participants) affected by a BSI related to a $K$. pneumoniae strain $(\mathrm{E}=$ exposure); comparison according to the presence or not of carbapenem resistance ( $\mathrm{C}=$ comparison); mortality as the main end point ( $\mathrm{O}=$ outcome).

\section{Literature Search}

All studies deemed eligible in the previous meta-analysis (literature search through 31 August 2016) [7] were considered for inclusion in the present IPD meta-analysis. A search update to 20 April 2018 through MEDLINE, EMBASE, CINAHL and Cochrane Database, using the same search strategy as in the previous meta-analysis (Supplementary Table S1), was performed with the help of a professional librarian. No geographical or language restrictions were applied.

After de-duplication, new records were screened for eligibility according to the aforementioned inclusion and exclusion criteria by two researchers (AG and SMP) in an independent fashion, and any potential disagreement was resolved through the intervention of a third investigator (SC).

Of note, all studies deemed eligible in the previous meta-analysis, both those that were selected and those that were not taken into account because of lack of feedback from the 
Table 1 Inclusion and exclusion criteria

\begin{tabular}{ll}
\hline Setting & $\begin{array}{l}\text { Cohort and case-control studies in adult populations hospitalized in acute-care } \\
\text { hospitals or intensive care units }\end{array}$ \\
\hline Inclusion criteria & Availability of mortality data for patients with CRKP and CSKP BSI \\
& Availability of patient-level information regarding: sex, age, Charlson Comorbidity Index, \\
& strain (CRKP versus CSKP), administered antibiotics (empirical and targeted) and their \\
& activity according to AST \\
& Studies published after 1994 \\
& Studies involving $<10$ patients per group \\
Exclusion criteria & Studies without a control group (e.g., only CRKP or only CSKP cases) or with an \\
& uninfected control group (e.g., colonized patients) \\
In case of several comparison groups, the one with the worst pattern of resistance regarding \\
CSKP (i.e., ESBL producers) was selected \\
Note on carbapenem- \\
CDC criteria were adopted: non-susceptibility to $\geq 1$ carbapenem or expression of a \\
resistance definition
\end{tabular}

$A S T$ antimicrobial susceptibility testing, BSI bloodstream infection, CDC Centers for Disease Control and Prevention, CRKP carbapenem-resistant $K$. pneumonia, CSKP carbapenem-susceptible $K$. pneumonia, ESBL extended-spectrum betalactamase

authors, were considered for inclusion (authors were contacted again).

\section{Data Extraction and Quality Assessment}

We contacted the authors of all selected studies (from the previous and the current search) via email to obtain the following de-identified patient-level data: age, sex, Charlson Comorbidity Index (CCI) score, agents used for empirical and targeted (according to antimicrobial susceptibility test [AST] results) treatment of CRKP and CSKP BSI, appropriateness of each agent used for empirical and targeted treatment of CRKP and CSKP BSI (appropriateness was defined when in vitro activity of the employed agent against the causative KP isolate was categorized as "susceptible" based on the clinical breakpoints employed in each study) and outcome (14-30 day or in-hospital mortality, depending on the end point employed in each e study). Two investigators (AEM and DRG) independently assessed the risk of bias of all included studies by using the Newcastle-Ottawa Quality Assessment Scale (NOS): observational studies with at least seven stars were categorized as having low risk of bias, those with five or six stars as medium risk of bias and those with fewer than four stars as high risk of bias [10]. Discrepancies were solved with the help of a third researcher (SC).

\section{Data Synthesis and Analysis}

We conducted a conventional two-stage IPD meta-analysis, an approach that automatically accounts for clustering of patients across the studies, by analyzing information from each study separately in the first stage [11].

Aggregated data from the initial step, wherein a logistic regression analysis, with mortality as dependent variable, was performed for each study, were then combined in the second step using a random effects technique pursuant to the DerSimonian and Laird procedure to estimate a summary OR with $95 \%$ CI [12].

Adjusted analyses were performed including the following variables in the logistic regression 
model: age (years), sex, Charlson Comorbidity Index score and publication year.

Heterogeneity was estimated using the $I^{2}$ statistic (values of $25 \%, 50 \%$ and $75 \%$, representing the thresholds of small, moderate and high levels of heterogeneity).

Results were graphically reported by mean of forest plot.

To investigate the presence of small study effects possibly associated with publication bias, a funnel plot with the pseudo 95\% CI for mortality was reported. In the absence of publication bias, the studies should be symmetrically distributed around the overall effect size. To test the funnel plot asymmetry, the regression-based Egger's test was performed.

Missing information was handled by means of complete case analysis.

Stata software (v16.0; StataCorp.) was used for statistical analysis.

\section{Outcomes}

The primary objective was to compare mortality between patients with CRKP and CSKP BSI through unadjusted (uOR) and adjusted (aOR) estimates in the overall cohort and in the following subgroups: (1) patients receiving inappropriate empirical therapy (no therapy or only inappropriate agents according to their in vitro activity); (2) patients receiving appropriate empirical therapy (at least one active drug in vitro); (3) patients receiving appropriate targeted therapy after inappropriate empirical therapy; (4) patients receiving appropriate targeted treatment.

We also conducted a secondary analysis in the CRKP BSI group, generating unadjusted and adjusted estimates for mortality carrying out the following comparison: appropriate targeted therapy with only one active agent (with or without inactive companion agents) vs. appropriate targeted therapy with at least two active drugs; appropriate targeted therapy with colistin plus any other active agent vs. appropriate targeted colistin monotherapy; appropriate targeted colistin plus an appropriate targeted aminoglycoside vs. appropriate targeted colistin monotherapy; appropriate targeted regimens (any) plus at least a carbapenem (inappropriate by definition) vs. appropriate targeted regimens (any) without carbapenem addition.

\section{Ethics}

Ethics committee approval was not required since the project used anonymized data and original studies had already received proper institutional review board approval.

\section{RESULTS}

\section{Overview of Data}

We obtained IPD data from 14 out of 28 eligible observational studies [13-26]: those not providing IPD information are listed in Supplementary Table S2. The entire selection process is depicted in Fig. 1.

The 14 included studies, whose main features are illustrated in Table 2, involved 2286 patients in the following countries: Italy (4), Greece (3), India (2), Israel (2) and USA (1) plus 2 multicenter international studies. Two were case-control studies and 12 were cohort studies (5 prospective and 7 retrospective). The time span of their conduction ranged from 2004 to 2016. The majority was represented by singlecenter studies (9 out of 14). Three studies addressed BSI infections only in intensive care unit (ICU) patients $[14,16,23]$. Of note, two studies were conducted in overlapping cohorts of onco-hematologic subjects $[22,26]$ : to the purposes of the present IPD work, their patient data were de-duplicated to provide unique cases.

A summary of CRKP and CSKP patient data is provided in Table 3 . Overall, sufficient therapeutic data for inclusion were available for $1952 / 2284$ patients with BSI enrolled in the included studies (1093 and 859 in the CRKP and CSKP groups, respectively). The mean age was 60 years in both groups. The CCI was higher in the CRKP group (median value 4 versus 3 ). Male sex was prevalent in both CRKP $(686 / 1058$, $64.8 \%)$ and CSKP patients (472/793, 59.5\%). 

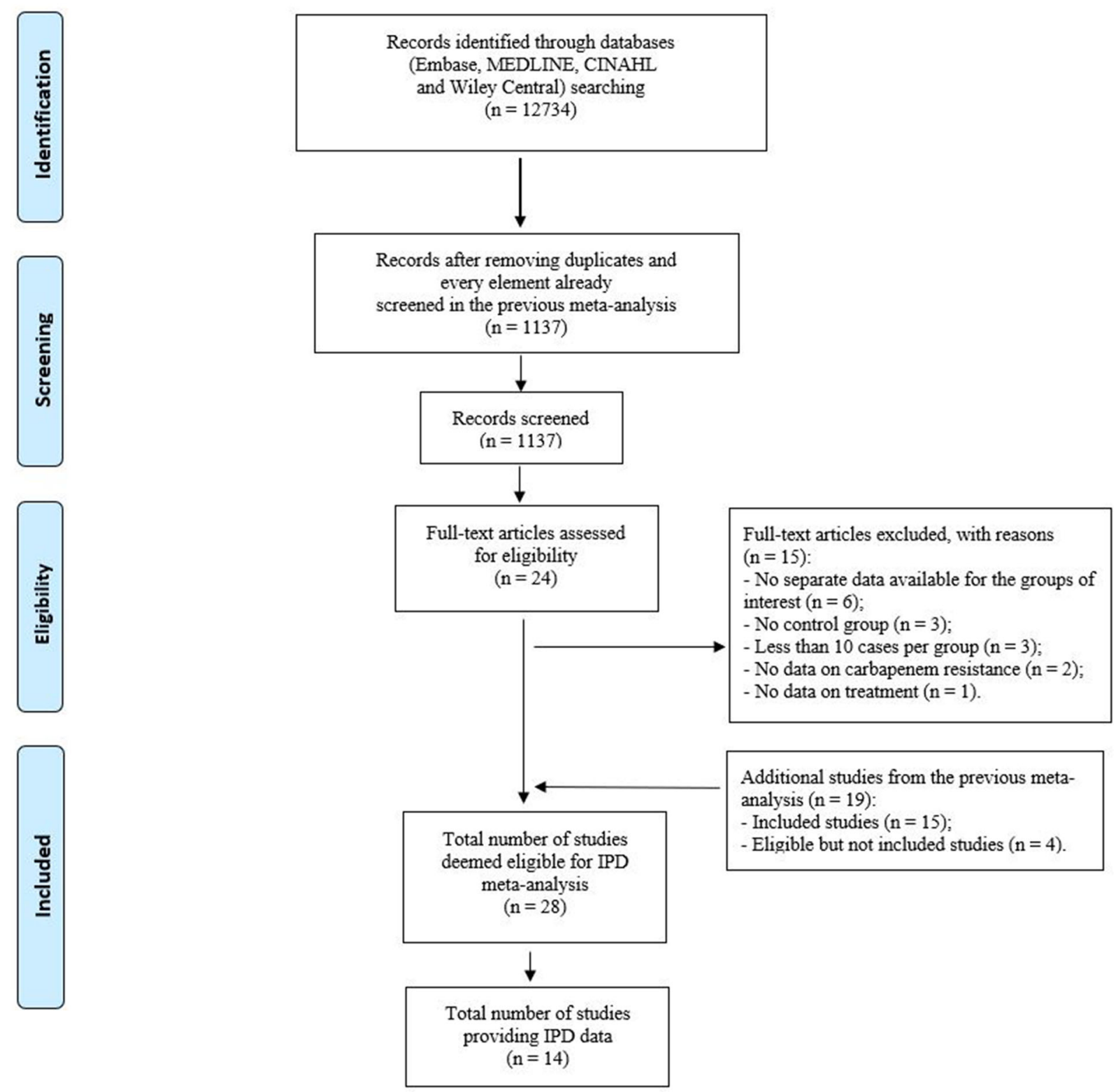

Fig. 1 Flowchart of included studies through literature search. IPD individual patient data

An appropriate empirical therapy was less frequent in CRKP $(353 / 1,061,33.3 \%)$ than in CSKP $(495 / 840,58.9 \%)$ patients (OR 0.35; 95\% CI 0.29-0.42). Most appropriate empirical therapies included only one in vitro active agent, with or without inactive companion agents (220/345 [63.8\%] in CRKP patients and 354/483 [73.3\%] in CSKP patients). In CRKP patients, colistin was the most frequent only active empirical drug $(122 / 220,54.5 \%)$, whereas in
CSKP patients the most frequent only active agent was a carbapenem (139/354, 39.2\%). No therapy administration before microbiologic diagnosis was the reason for inappropriate empirical therapy in $36.7 \%(260 / 708)$ and $16.9 \%(142 / 840)$ of CRKP and CSKP patients, respectively.

Not all patients received an appropriate targeted treatment. In more detail, an appropriate targeted therapy was administered in 706/896 


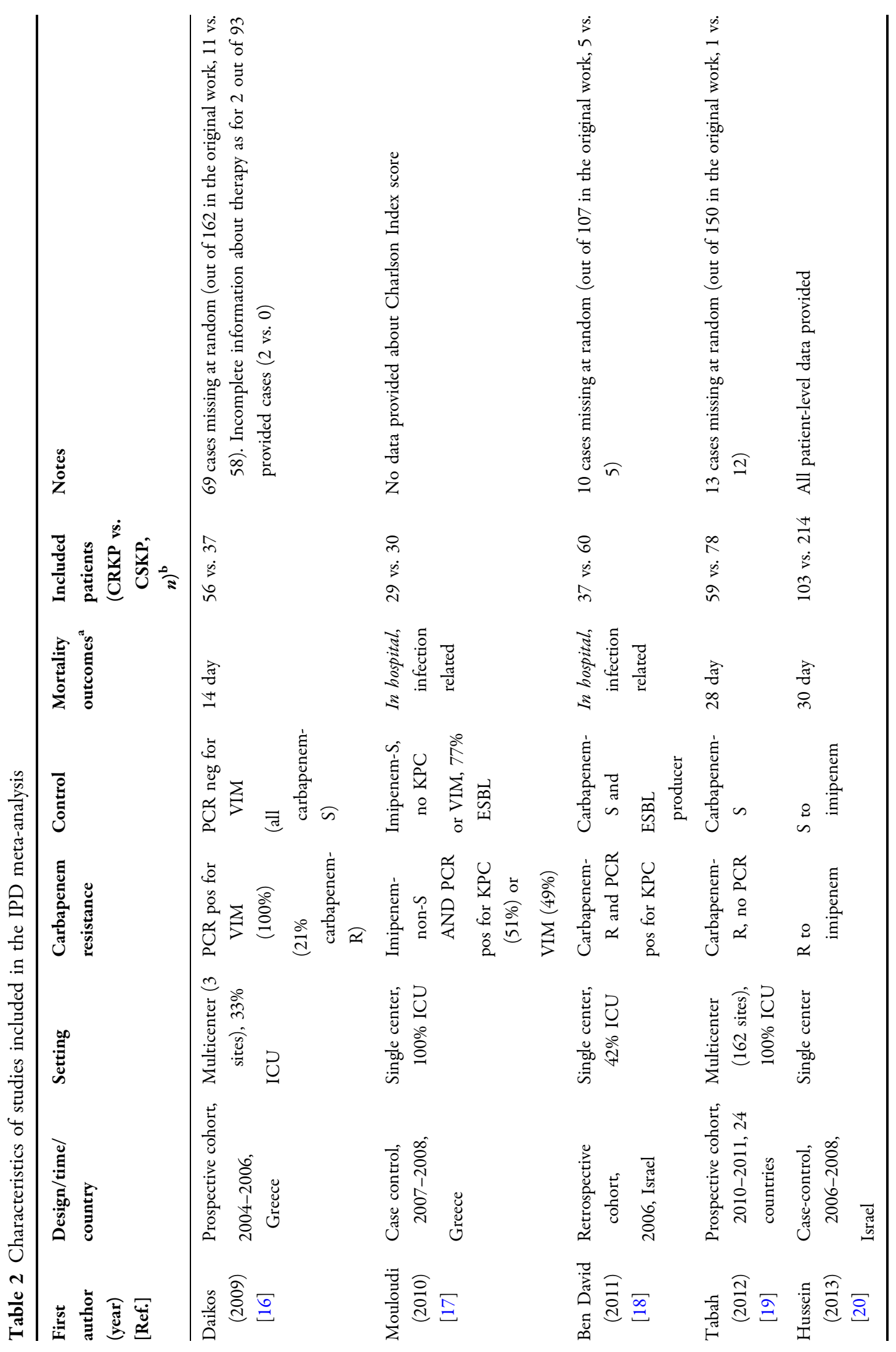




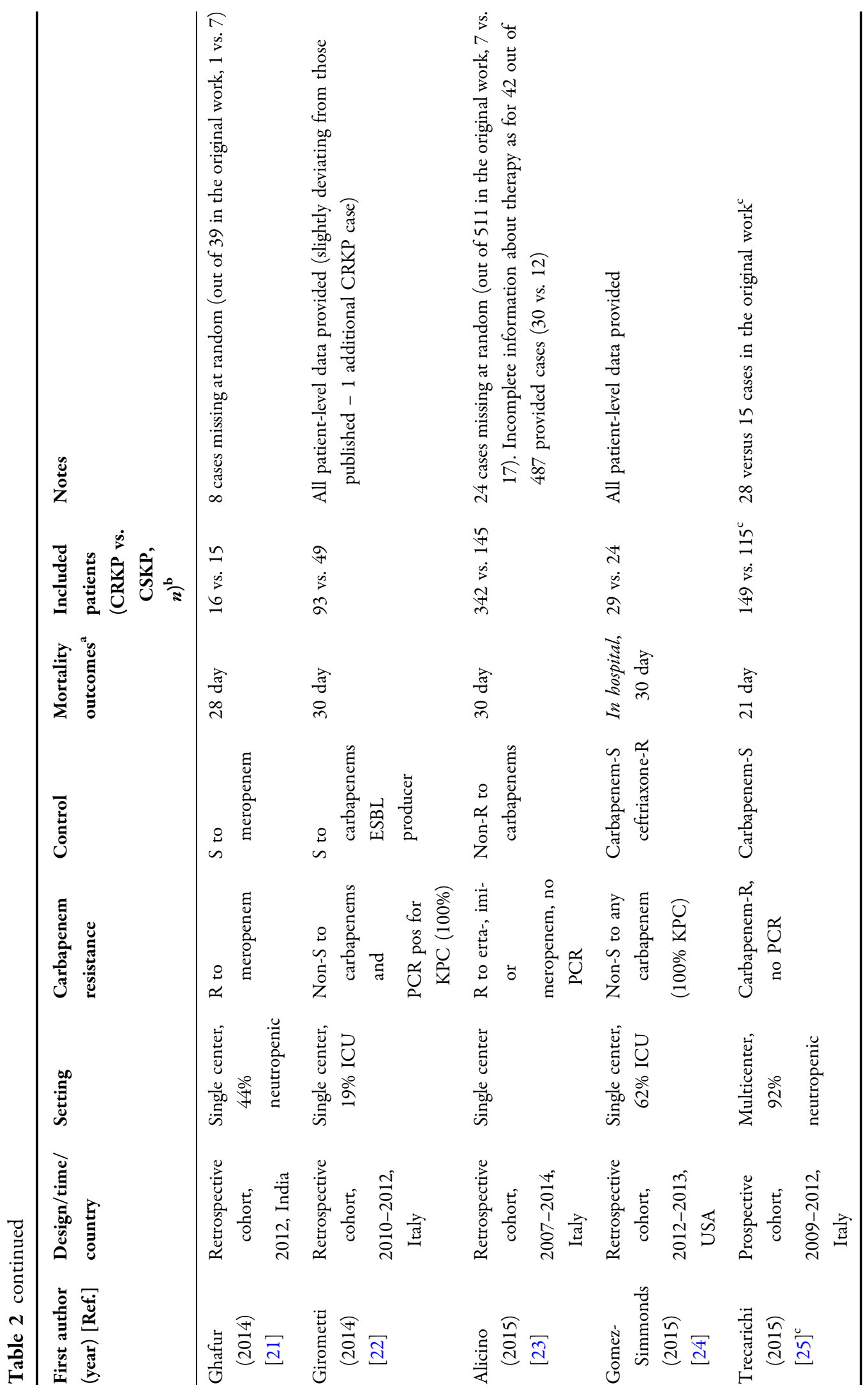




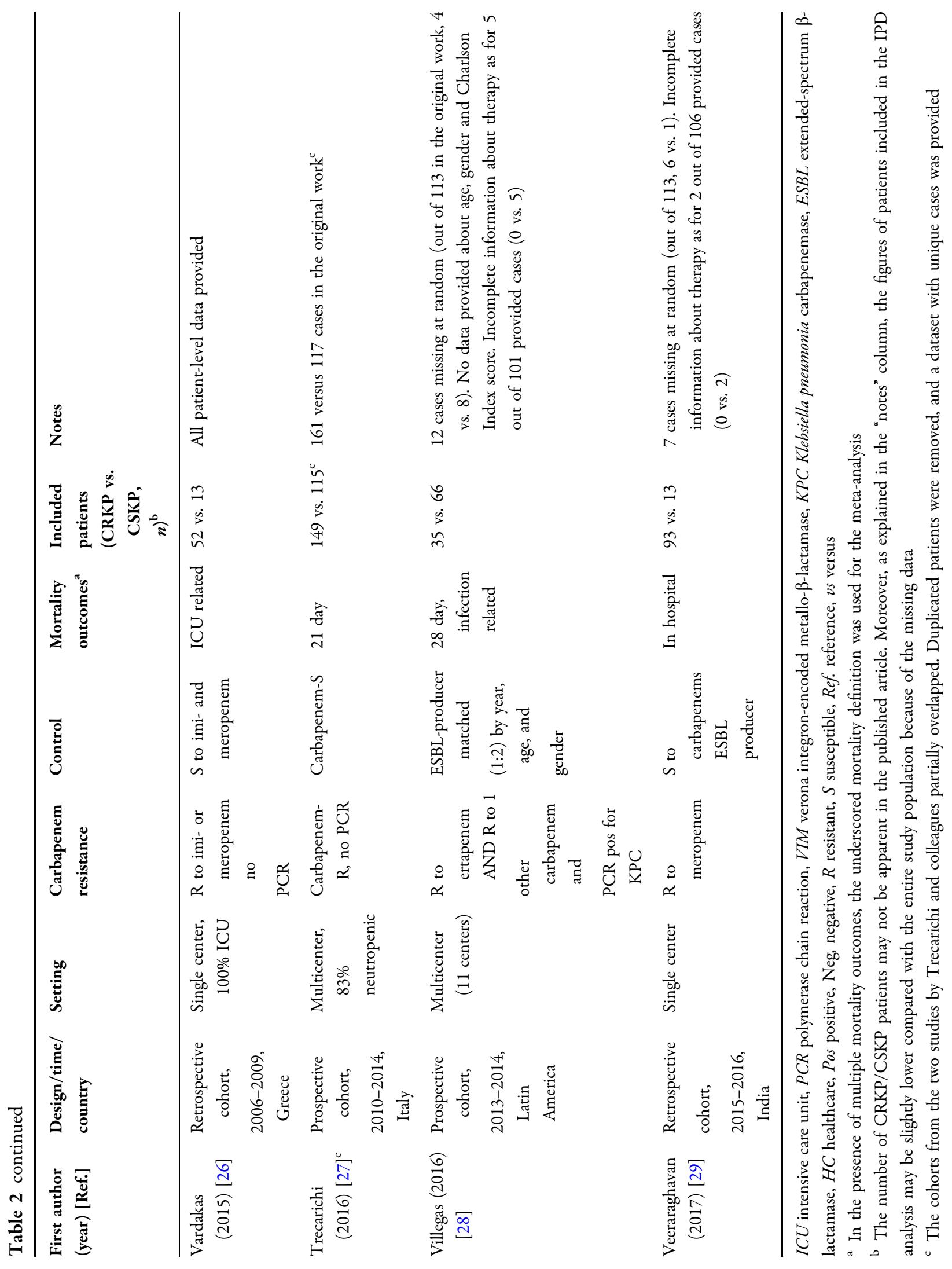


Table 3 Summary of the main individual data regarding CRKP and CSKP patients

\begin{tabular}{|c|c|c|c|c|c|c|}
\hline \multirow[t]{2}{*}{ Variable } & \multicolumn{3}{|c|}{ CRKP group $(n=1093)$} & \multicolumn{3}{|c|}{ CSKP group $(n=859)$} \\
\hline & $\begin{array}{l}\text { Valid } \\
\text { sample }\end{array}$ & Missing & Measure & $\begin{array}{l}\text { Valid } \\
\text { sample }\end{array}$ & Missing & Measure \\
\hline Age, mean years $(\mathrm{SD})$ & 1058 & 35 & $59.7(18.3)$ & 793 & 66 & $60.4(18.4)$ \\
\hline Sex (male, \%) & 1058 & 35 & $686(64.8)$ & 793 & 66 & $472(59.5)$ \\
\hline Charlson Comorbidity Index, median (IQR) & 1029 & 64 & $4(2-5)$ & 763 & 96 & $3(2-5)$ \\
\hline Active empirical therapy (\%) & 1061 & 32 & $\begin{array}{l}353 \\
(33.3)^{\mathrm{a}}\end{array}$ & 840 & 19 & $495(58.9)^{\mathrm{a}}$ \\
\hline $\begin{array}{l}\text { Active empirical therapy based on } 1 \mathrm{drug} \text {, alone or } \\
\text { in combination with inactive agents }(\%)\end{array}$ & 345 & $\begin{array}{l}8 \text { (out } \\
\text { of } \\
353)\end{array}$ & $220(63.8)$ & 483 & $\begin{array}{l}12 \text { (out } \\
\text { of } \\
495)\end{array}$ & $354(73.3)$ \\
\hline $\begin{array}{l}\text { Most frequent active drug when only one empirical } \\
\text { agent was active } \\
(n, \%)\end{array}$ & 220 & & $\begin{array}{l}\text { Colistin } \\
\qquad \begin{array}{l}(122 \\
54.5)\end{array}\end{array}$ & 483 & & $\begin{array}{l}\text { Carbapenem } \\
\text { agent }^{\text {b }} \\
(139,39.2)\end{array}$ \\
\hline Most frequent inactive empirical therapy & 708 & & $\begin{array}{l}\text { No } \\
\text { therapy } \\
(260,36.7)\end{array}$ & 840 & & $\begin{array}{l}\text { No therapy } \\
\quad(142,16.9)\end{array}$ \\
\hline No. of active empirical antibiotics, median (IQR) & 352 & $\begin{array}{l}1 \text { (out } \\
\text { of } \\
353)\end{array}$ & $1(1-2)$ & 480 & $\begin{array}{l}15 \text { (out } \\
\text { of } \\
495)\end{array}$ & $1(1-2)$ \\
\hline Active targeted therapy (\%) & 896 & 60 & $\begin{array}{c}706 / 896 \\
(78.8)\end{array}$ & 781 & 8 & $\begin{array}{r}678 / 781 \\
(86.8)\end{array}$ \\
\hline $\begin{array}{l}\text { Active targeted therapy based on } 1 \mathrm{drug} \text {, alone or in } \\
\text { combination with inactive agents }(\%)\end{array}$ & 896 & & $364(40.6)$ & 781 & & $543(69.5)$ \\
\hline $\begin{array}{l}\text { Most frequent active drug when only one targeted } \\
\text { agent was active }\end{array}$ & 364 & & $\begin{array}{l}\text { Colistin } \\
(225,61.8)\end{array}$ & 543 & & $\begin{array}{l}\text { Carbapenem } \\
\text { agent }^{c}\end{array}$ \\
\hline$(n, \%)$ & & & & & & $(309,56.9)$ \\
\hline No. of active targeted antibiotics, median (IQR) & 896 & & $2(1-2)$ & 781 & & $1(1-1)$ \\
\hline Mortality (\%) & 1093 & & $466(42.6)$ & 859 & & $231(26.9)$ \\
\hline
\end{tabular}

BSI bloodstream infection, CRKP carbapenem-resistant Klebsiella pneumonia, CSKP carbapenem-susceptible Klebsiella pneumoniae, IQR interquartile range, $S D$ standard deviation

${ }^{a}$ CRKP-infected patients were less likely to receive active empirical therapy than CSKP-infected subjects (OR 0.35; $95 \%$ CI $0.29-0.42)$

b Meropenem 76, ertapenem 38, imipenem 25; as single agent piperacillin/tazobactam was the one most used (93, 26.3\%)

c Meropenem 137, ertapenem 126, imipenem 46 
(78.8\%) and 678/781 (86.8\%) CRKP and CSKP patients, respectively (the denominators did not include subjects who died before receiving targeted drugs). The most appropriate targeted therapies included only one in vitro active agent, with or without inactive companion agents (364 [40.6\%] in CRKP patients and 543 [69.5\%] in CSKP patients). In CRKP patients, colistin was the most frequent active empirical monotherapy $(225 / 364,61.8 \%)$, whereas in CSKP patients the most frequent active monotherapy was a carbapenem (309/543, $56.9 \%)$. When considering combination regimens including at least two active anti-CRKP agents as appropriate targeted treatment (332/ $686,48.4 \%)$, colistin plus tigecycline was the most common scenario $(97 / 322,30.1 \%)$, followed by colistin plus gentamicin (45/322, $13.9 \%)$.

\section{Primary Analyses}

In the included studies, mortality was assessed as follows: in hospital (5 studies), 30 day (3 studies), 28 day (3 studies), 21 day (2 studies) and 14 day (1 study).

Overall, crude mortality was as follows: $42.6 \%$ in CRKP patients (466/1093); $26.9 \%$ in CSKP patients (231/859). Mortality was higher in CRKP than CSKP patients (uOR 2.18; 95\% CI $1.60-2.90 ; I^{2}=47.7 \%$; Supplementary Fig S1). This result was confirmed in the adjusted model $\left(\right.$ aOR 2.17; 95\% CI 1.56-3.04; $I^{2}=44.1 \%$; Fig. 2).

When considering empirical therapy, mortality was higher in patients with CRKP than CSKP BSI, in both the subgroup of inappropriate (uOR 1.95; 95\% CI, 1.42-2.69; $I^{2}=0$ ) and of appropriate empirical therapy (uOR 2.33; 95\% CI $\left.1.64-3.31 ; I^{2}=13 \%\right)$. This unfavorable effect of carbapenem resistance did not modify between the two subgroups, as shown by the test for interaction $(p=0.46$, Supplementary Fig. S2). Similar results were observed in adjusted subgroup models: inappropriate empirical therapy (aOR 1.75; 95\% CI 1.24-2.47; $I^{2}=0$ ); appropriate empirical therapy (aOR 2.66; 95\% CI 1.70-4.16; $\left.I^{2}=16 \%\right)$. The test for interaction was also not significant ( $p=0.15$; Fig. 3 ).
There was no statistical difference in mortality between CRKP and CSKP patients also in the subgroup of patients with inappropriate empirical therapy followed by appropriate targeted therapy (sufficient data from 7 studies): aOR 1.53 (95\% CI 0.87-2.68; $I^{2}=0$; Fig. 4); the uOR was the same, 1.59 (95\% CI 0.95-2.64; $I^{2}=0$ ). Finally, mortality was higher in CRKP than CSKP patients in the subgroup of patients receiving appropriate targeted therapy in both the unadjusted (uOR 2.22, 95\% CI 1.45-3.43; $I^{2}=58 \% ;$ Supplementary Fig. S3) and the adjusted models (aOR 2.21; 95\% CI 1.36-3.59; $I^{2}=58 \%$; Fig. 5).

\section{Secondary Analyses}

Secondary analyses were limited to the subgroup of CRKP patients. Their results are summarized in Table 4 . As shown in the table, albeit with the limitation of the reduced number of studies and participants in secondary analyses, no statistically significant associations were observed in any of the comparisons: (1) appropriate targeted treatment with only one active agent (with or without inactive companion agents) vs. appropriate targeted therapy with at least two active drugs (unadjusted model: uOR 0.91 ; $95 \%$ CI $0.58-1.43, I^{2}=36.9 \%$; adjusted model: aOR 0.78; 95\% CI 0.43-1.45, $I^{2}=53.3 \%$, Supplementary Fig. S4); (2) appropriate targeted therapy with colistin plus any other active agent vs. appropriate targeted colistin monotherapy (unadjusted model: uOR 1.13; 95\% CI 0.70-1.83, $I^{2}=36.9 \%$; adjusted model: aOR $1.24 ; 95 \%$ CI $0.58-266, I^{2}=43.3 \%$, Supplementary Fig. S5); (3) appropriate targeted colistin plus an appropriate targeted aminoglycoside vs. appropriate targeted colistin monotherapy (sufficient data just from 3 studies; unadjusted model: uOR 0.74 ; $95 \%$ CI $0.34-1.61, I^{2}=10 \%$; adjusted model: aOR 0.6; $95 \%$ CI $0.26-1.56, I^{2}=47 \%$, Supplementary Fig. S6); (4) appropriate targeted regimens (any) plus at least a carbapenem (inappropriate by definition) vs. appropriate targeted regimens (any) without carbapenem addition (data only from 4 studies; unadjusted model: uOR 1.40; 95\% CI $0.26-7.66, I^{2}=73 \%$; adjusted analysis: 


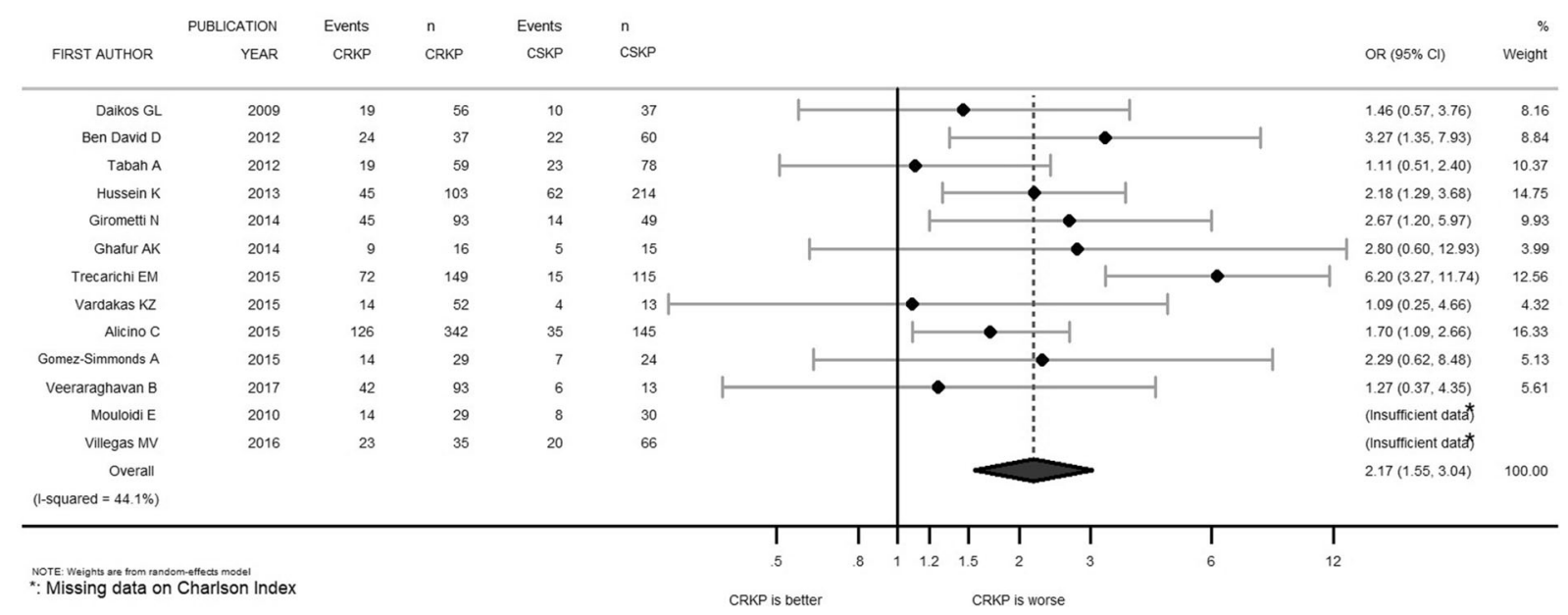

Fig. 2 Forest plot depicting adjusted mortality between CRKP BSI and CSKP BSI patients. BSI bloodstream infection, CRKP carbapenem-resistant Klebsiella pneumoniae, CSKP carbapenem-susceptible Klebsiella pneumoniae

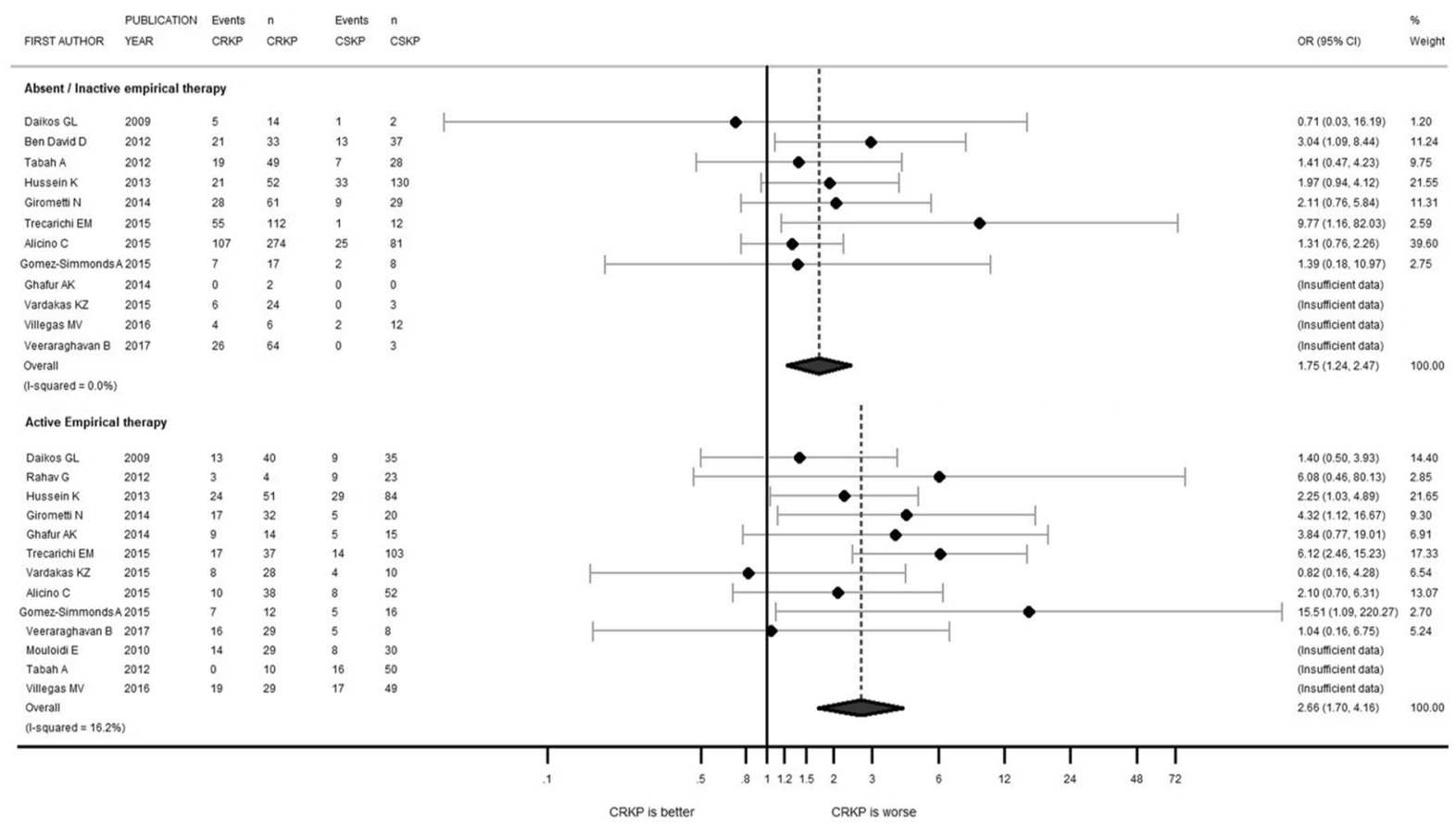

Fig. 3 Forest plot depicting adjusted mortality between CRKP BSI and CSKP BSI patients by considering the presence and activity of empirical therapy. BSI bloodstream infection, CRKP carbapenem-resistant Klebsiella

aOR 1.37; 95\% CI 0.20-9.38, $I^{2}=71 \%$; Supplementary Fig. S7). pneumonia, CSKP carbapenem-susceptible Klebsiella pneumoniae. Note: The study by Mouloudi and colleagues [17] did not present patients with no or not active (inappropriate) empirical therapy

\section{Risk of Bias Assessment}

The funnel plot did not reveal consistent asymmetry, and this was confirmed by the 


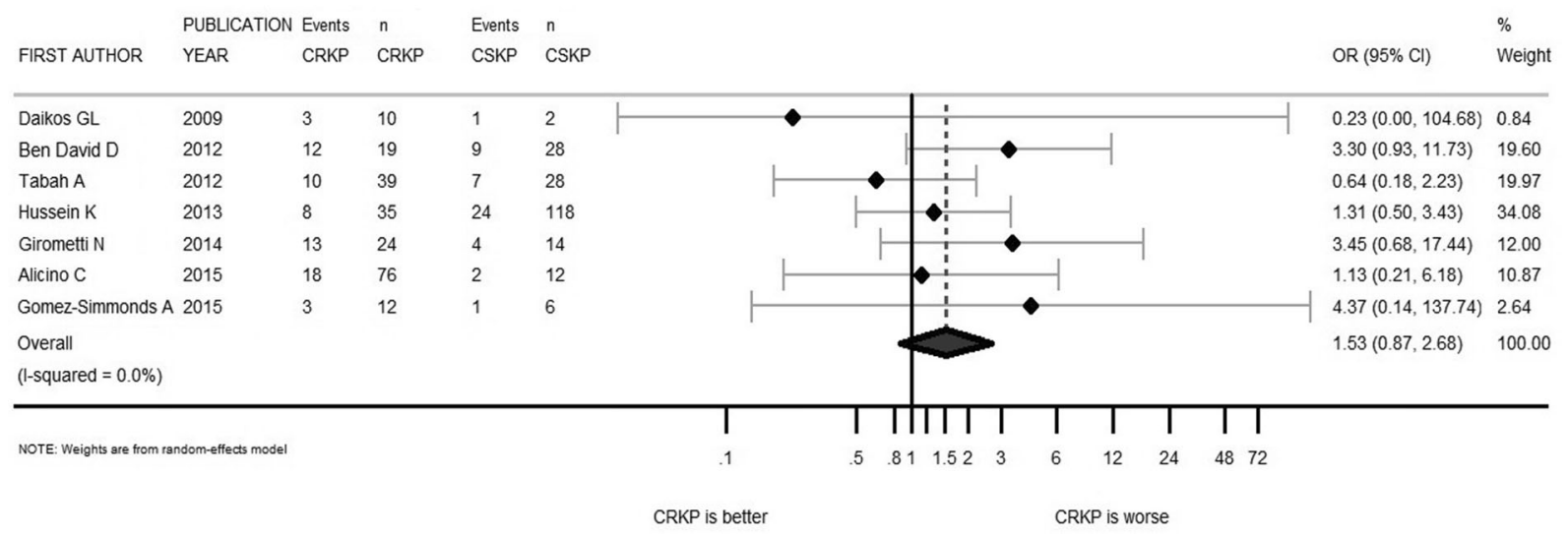

Fig. 4 Forest plot depicting adjusted mortality between CRKP BSI and CSKP BSI patients when absent/inactive initial treatment was followed by targeted therapy. BSI bloodstream infection, CRKP carbapenem-resistant Klebsiella pneumonia, CSKP carbapenem-susceptible Klebsiella pneumoniae

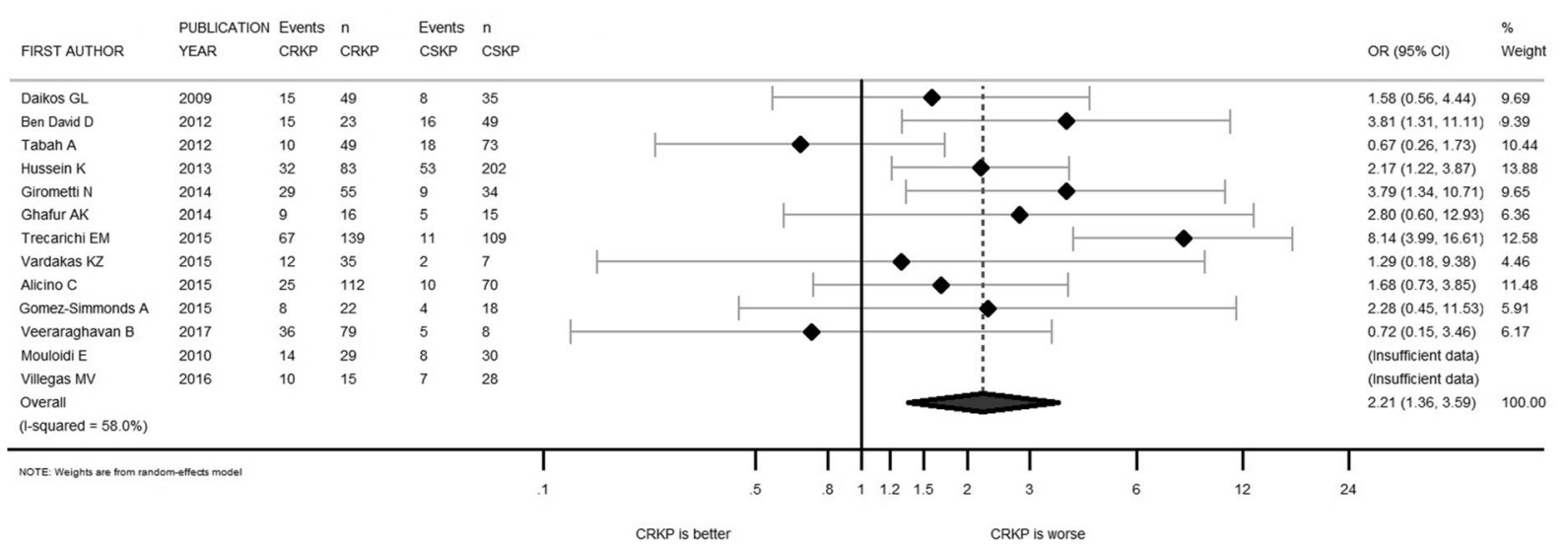

Fig. 5 Forest plot depicting adjusted mortality between CRKP BSI and CSKP BSI patients when active targeted treatment was employed. BSI bloodstream infection,

Egger's test with $p=0.52$ (Supplementary Fig. S8).

In Supplementary Table S4, the quality assessment is illustrated. The overall risk of bias was moderate in half of the studies and low in the other half.

\section{DISCUSSION}

The present study, to our knowledge the first IPD meta-analysis comparing the mortality of CRKP and CSKP BSI, was conceived in the wake
CRKP carbapenem-resistant Klebsiella pneumoniae, CSKP carbapenem-susceptible Klebsiella pneumoniae

of a previous non-IPD meta-analysis conducted by Kohler and colleagues [7]. Our study followed this approach to focus only upon a specific type of infection (to reduce clinical heterogeneity) and upon a given pathogen (KP, which is the paradigmatic model of carbapenem-resistance among Enterobacterales [27-29]).

In the previous meta-analysis, a higher mortality risk in CRKP BSI than in CSKP BSI was registered not only in the overall cohort (uOR $2.66,95 \%$ CI 1.83-3.87), but also in the subgroup of patients receiving appropriate 
empirical therapy (uOR 2.21, 95\% CI 1.29-3.81). Notably, the persistence of such a difference in mortality in this subgroup seemed to suggest that factors other than a different proportion of appropriate empirical therapy (expected to be lower in CRKP patients) were involved in explaining the increased mortality observed in CRKP vs. CSKP patients. There are many plausible, non-mutually exclusive explanations [30, 31], and the most important are the following: (1) increased burden of comorbidities in CRKP compared to CSKP patients [32]; (2) increased virulence of CRKP [33]; (3) increased toxicity and/or suboptimal efficacy of active agents against CRKP vs. those used for CSKP (for

Table 4 Secondary analyses regarding only patients with CRKP BSI

\begin{tabular}{|c|c|c|}
\hline Outco & $\begin{array}{l}\text { uOR }(95 \% \\
\text { CI) }\left[\mathbf{I}^{2}\right]\end{array}$ & $\begin{array}{l}\underset{\mathrm{aOR}}{\left[\mathrm{I}^{2}\right]}(95 \% \mathrm{CI}) \\
\end{array}$ \\
\hline $\begin{array}{l}\text { Targeted } \\
\text { monotherapy vs. } \\
\text { targeted } \\
\text { combination } \\
\text { regimens }\end{array}$ & $\begin{array}{l}0.91 \\
\quad(0.58-1.43) \\
{[36.9 \%]}\end{array}$ & $\begin{array}{l}0.78(0.43-1.45) \\
{[53.3 \%]} \\
\quad \text { Suppl. } \\
\text { Figure 4) }\end{array}$ \\
\hline $\begin{array}{l}\text { Targeted colistin plus } \\
\text { another active drug } \\
\text { vs. colistin alone }\end{array}$ & $\begin{array}{l}1.13 \\
\quad(0.70-1.83) \\
{[9 \%]}\end{array}$ & $\begin{array}{l}1.24(0.58-2.66) \\
{[43.3 \%]} \\
\text { (Suppl. } \\
\text { Figure 5) }\end{array}$ \\
\hline $\begin{array}{l}\text { Targeted colistin plus } \\
\text { an active } \\
\text { aminoglycoside } \\
\text { agent vs. colistin } \\
\text { alone }\end{array}$ & $\begin{array}{l}0.74 \\
\quad(0.34-1.61) \\
\quad[10 \%]\end{array}$ & $\begin{array}{c}0.63(0.26-1.56) \\
{[47 \%] \text { (Suppl. }} \\
\text { Figure 6) }\end{array}$ \\
\hline $\begin{array}{l}\text { Additional } \\
\text { carbapenem to a } \\
\text { targeted regimen vs. } \\
\text { active regimens } \\
\text { without } \\
\text { carbapenem agents }\end{array}$ & $\begin{array}{l}1.40 \\
(0.26-7.66) \\
{[73 \%]}\end{array}$ & $\begin{array}{c}1.37(0.20-9.38) \\
{[71 \%] \text { (Suppl. }} \\
\text { Figure } 7)\end{array}$ \\
\hline
\end{tabular}

$a O R$ adjusted odds ratio, $B S I$ bloodstream infection, CRKP carbapenem-resistant Klebsiella pneumonia, uOR unadjusted odds ratio example, in most studies [13-26] colistin, which has inherent pharmacokinetics and toxicity issues [34, 35], was the most used appropriate therapy for CRKP, whereas for CSKP the most used appropriate therapy was a carbapenem) [36].

In the present IPD-MA, we tried to bring more insights into the discussion about all these possible additional explanations. Regarding the first of them (increased burden of comorbidities in CRKP patients), the difference in mortality between CSKP and CRKP patients was retained even when adjusting for CCI, not only in the overall IPD-MA population, but also in the subgroups stratified for appropriateness of therapy. In our opinion, this suggests that a similar burden of comorbidities is unable to cancel the difference in mortality between CRKP and CSKP patients we observed in this study, that is, at least another additional/alternative factor must be involved.

Regarding the second possible explanation (increased virulence of CRKP), it cannot be addressed in our analysis. Nonetheless, it should be noted that in the presence of a large difference in virulence of CRKP vs. CSKP, the largest difference in mortality would have reasonably been expected in inappropriately treated patients (i.e., no activity of therapy in either CRKP or CSKP BSI). Conversely, our results seem to support (with the major limitation of a very limited adjustment for comorbidities and acute phase conditions) a more decisive effect of therapy (i.e., of the increased toxicity and/or suboptimal efficacy of active agents against CRKP vs. those used for CSKP). Indeed, although in both the subgroup of patients receiving inappropriate empirical therapy and the subgroup of patients receiving appropriate targeted therapy after inappropriate empirical therapy mortality was higher in the CRKP than in the CSKP group (aOR 1.75 and 1.53, respectively), this impact of carbapenem resistance on mortality seemed to be less pronounced than in the subgroup of patients receiving appropriate empirical therapy (aOR 2.66). In other words, when an appropriate therapy had been administered since the onset of symptoms (i.e., when the favorable impact of an appropriate therapy on mortality was expected to be the highest, 
since there was no unfavorable effect of delayed administration), we registered the largest difference in mortality between CRKP BSI and CSKP BSI, which could be in line with the supposed reduced efficacy/increased toxicity of appropriate treatment for CRKP BSI (e.g., polymyxins) vs. those for CSKP (e.g., carbapenems). Unfortunately, there were not sufficient numbers of CRKP and CSKP patients treated with the same appropriate empirical drug according to their in vitro susceptibility (e.g., colistin), which could have provided even more homogeneous subgroups to further delve into this aspect, which herein still remains largely speculative.

The present work has other important limitations. For instance, our secondary analyses comparing different treatment options for CRKP BSI were underpowered in the light of the retrieved number of IPD-MA participants in the subgroup of CRKP. Consequently, unfortunately no firm conclusion can stem from the present work about the effectiveness of combination regimens vs. monotherapy for CRKP BSI (both in general and when considering only colistin-based therapies) as well as about the possible role of the addition of carbapenems. It should also be noted that the present work inherited some limitations of the previous nonIPD meta-analysis [7], e.g., the inclusion of only observational studies, since no prospective randomized trials were retrieved, thereby the ones included were characterized by relevant distortions that can be corrected, but not completely eliminated, through appropriate statistical techniques [37]. Another important limitation is the relatively low number of patient-level variables that were available from the original datasets; the following potentially important predictors were missing from the data analysis: the exact mechanisms of resistance, source of bacteremia (and its control through related measures, such as central line removal), number of days elapsed from infection to appropriate therapy, severity of clinical presentation and precise antibiotic dosages. Moreover, it should be noted that drug activity in vitro is not always a synonym of appropriateness of therapy [38]. Eventually, the time span of included studies did not extend beyond 2016: the impact of newer anti-CRKP drugs could not be evaluated, so most anti-CRKP therapies were colistinbased.

However, important strengths of our work should be highlighted, related to the IPD design, which allows to: (1) identify overlapping sets of patients to avoid duplicated data; (2) provide estimates adjusted for baseline factors consistently across the studies when previously only unadjusted effect sizes had been produced; (3) give meta-analytic results for specific subgroups; (4) prevent ecologic bias by avoiding study-level confounding that may affect aggregate data analysis [12].

\section{CONCLUSIONS}

In conclusion, the present, first IPD-MA comparing the mortality of CRKP and CSKP BSI further confirms the unfavorable impact of carbapenem resistance observed in previous observational studies and non-IPD meta-analyses [5-7] and provides some important additional insights to enrich our understanding of the underlying causative reasons, especially concerning the possible effect of the different classes of appropriate agents available for CRKP and CSKP infections. From this standpoint, we shall greatly welcome possible future IPD-MA also including CRKP participants treated with novel, possibly more efficacious and less toxic anti-CRKP agents, which, in our opinion, could be the ultimate missing piece to solve this complex, long-standing puzzle.

\section{ACKNOWLEDGEMENTS}

We are very grateful to the following researchers for sharing their de-identified datasets: Dr. C. Alicino, Prof. G.L. Daikos; Prof. A.K. Ghafur; Prof. K. Hussein; Prof. G. Rahav; Prof. E. Roilides; Prof. J.F. Timsit; Prof. E.M. Trecarichi; Prof. A.C. Uhlemann; Prof. K.Z. Vardakas; Prof. B. Veeraraghavan; Prof. P. Viale; Prof. M.V. Villegas. Moreover, we are indebted to Elizabeth Uleryk (E.M. Uleryk Consulting, Mississauga, ON, Canada) for performing the literature search. 
Funding. No funding or sponsorship was received for this study or publication of this article.

Authorship. All named authors meet the International Committee of Medical Journal Editors (ICMJE) criteria for authorship for this article, take responsibility for the integrity of the work as a whole, and have given their approval for this version to be published.

Authorship contributions. AEM: Conceptualization, Methodology, Data Curation, Writing-Original Draft, Writing-Review and Editing. SC: Investigation, Data Curation, Writing-Review and Editing. AG: Investigation, Writing-Review and Editing AS: Formal analysis. CA: Writing-Review and Editing. $\mathrm{KH}$ : Writing-Review and Editing. EMT: WritingReview and Editing. PV: Writing-Review and Editing. JFT: Writing-Review and Editing. BV: Writing-Review and Editing. MVV: WritingReview and Editing. FR: Writing-Review and Editing. GLD: Writing-Review and Editing. KZV: Writing-Review and Editing. ER: Writing-Review and Editing. ACV: Writing-Review and Editing. AKG: Writing-Review and Editing. SMP: Investigation, Writing-Review and Editing. MB: Writing-Review and Editing. PPK: Writing-Review and Editing. DRG: Conceptualization, Methodology, Supervision. All authors discussed the results, commented on the manuscript and have approved the final version of the paper.

Collaborators. Debby Ben David, Maddalena Giannella, Nicolò Girometti, Angela Gomez-Simmonds, Evgenia Gouridou, Elias Iosifidis, Alice Joan Mathuram, Andrea Orsi, Livio Pagano, Christian Pallares Gutiérrez, Mina Psichogiou, Stephane Ruckly, Michael Samarkos, Alexis Tabah, Mario Tumbarello.

Disclosures. Outside the submitted work, Alberto E. Maraolo reports honoraria from Nordic Pharma; Abdul K. Ghafur received lecture fees, advisory fees, travel grants, research grants from MSD, Pfizer, Astellas, BioMerieux, Cipla, Wockhardt, Bharath serum, Natco, Mylan; Matteo Bassetti funding for scientific advisory board participation, travel and for speaking from Angelini, AstraZeneca, Basilea, Bayer, BioMerieux, Cidara, Correvio, Cubist, Menarini, Molteni, Merck Sharp and Dohme, Nabriva, Paratek, Pfizer, Roche, Shionogi, Tetraphase, ThermoFisher, and The Medicine Company; Matteo Bassetti is also a member of the journal's Editorial Board; Daniele R. Giacobbe reports honoraria from Stepstone Pharma $\mathrm{GmbH}$ and unconditional grants from Merck Sharp and Dohme Italia and Correvio Italia. Silvia Corcione, Adriano Grossi, Alessio Signori, Cristiano Alicino, Khetam Hussein, Enrico M. Trecarichi, Pierluigi Viale, Jean-François Timsit, Balaji Veeraraghavan, Maria V. Villegas, Galia Rahav, George L. Daikos, Konstantinos Z. Vardakas, Emmanuel Roilides, Anne-Catrin Uhlemann, Simone Mornese Pinna, Philipp P. Kohler have no competing interest to declare.

Compliance with Ethics Guidelines. This article is based on previously conducted studies and does not contain any studies with human participants or animals performed by any of the authors.

Data Availability. The datasets generated during and/or analysed during the current study are available from the corresponding author on reasonable request.

Open Access. This article is licensed under a Creative Commons Attribution-NonCommercial 4.0 International License, which permits any non-commercial use, sharing, adaptation, distribution and reproduction in any medium or format, as long as you give appropriate credit to the original author(s) and the source, provide a link to the Creative Commons licence, and indicate if changes were made. The images or other third party material in this article are included in the article's Creative Commons licence, unless indicated otherwise in a credit line to the material. If material is not included in the article's Creative Commons licence and your intended use is not permitted by statutory regulation or exceeds the permitted use, you will need to obtain permission directly from the copyright holder. To view a copy of this licence, 
visit http://creativecommons.org/licenses/bync/4.0/.

\section{REFERENCES}

1. Suay-García B, Pérez-Gracia MT. Present and future of carbapenem-resistant enterobacteriaceae (CRE) Infections. Antibiotics (Basel). 2019;8:E122.

2. Bartsch SM, McKinnell JA, Mueller LE, et al. Potential economic burden of carbapenem-resistant Enterobacteriaceae (CRE) in the United States. Clin Microbiol Infect. 2017;23(48):e9-48.e16.

3. Stewardson AJ, Marimuthu K, Sengupta S, et al. Effect of carbapenem resistance on outcomes of bloodstream infection caused by Enterobacteriaceae in low-income and middle-income countries (PANORAMA): a multinational prospective cohort study. Lancet Infect Dis. 2019;19:601-10.

4. Tacconelli E, Carrara E, Savoldi A, et al. Discovery, research, and development of new antibiotics: the WHO priority list of antibiotic-resistant bacteria and tuberculosis. Lancet Infect Dis. 2018;18: 318-27.

5. Li Y, Li J, Hu T, et al. Five-year change of prevalence and risk factors for infection and mortality of carbapenem-resistant Klebsiella pneumoniae bloodstream infection in a tertiary hospital in North China. Antimicrob Resist Infect Control. 2020;9:79.

6. Martin A, Fahrbach K, Zhao Q, et al. Association Between Carbapenem Resistance and Mortality Among Adult, Hospitalized Patients With Serious Infections Due to Enterobacteriaceae: Results of a Systematic Literature Review and Meta-analysis. Open Forum Infect Dis. 2018; 5: ofy150

7. Kohler PP, Volling C, Green K, et al. Carbapenem resistance, initial antibiotic therapy, and mortality in Klebsiella Pneumoniae Bacteremia: a systematic review and meta-analysis. Infect Control Hosp Epidemiol. 2017;38:1319-28.

8. Riley RD, Lambert PC, Abo-Zaid G. Meta-analysis of individual participant data: rationale, conduct, and reporting. BMJ. 2010;340:c221.

9. Stewart LA, Clarke M, Rovers M, et al. Preferred reporting items for systematic review and metaanalyses of individual participant data: the PRISMAIPD Statement. JAMA. 2015;313:1657-65.

10. Wells GA, Shea B, O'Connell D, et al. (2014) The Newcastle-Ottawa Scale (NOS) for assessing the quality of nonrandomised studies in meta-analyses.
The Ottawa Hospital Research Institute. Available from: http://www.ohri.ca/programs/clinical_ epidemiology/oxford.asp

11. Burke DL, Ensor J, Riley RD. Meta-analysis using individual participant data: one-stage and two-stage approaches, and why they may differ. Stat Med. 2017;36:855-75.

12. DerSimonian R, Laird N. Meta-analysis in clinical trials. Control Clin Trials. 1986;7:177-88.

13. Daikos GL, Petrikkos $P$, Psichogiou $M$, et al. Prospective observational study of the impact of VIM-1 metallo-beta-lactamase on the outcome of patients with Klebsiella pneumoniae bloodstream infections. Antimicrob Agents Chemother. 2009;53:1868-73.

14. Mouloudi E, Protonotariou E, Zagorianou A, et al. Bloodstream infections caused by metallo-beta-lactamase/Klebsiella pneumoniae carbapenemase-producing K. pneumoniae among intensive care unit patients in Greece: risk factors for infection and impact of type of resistance on outcomes. Infect Control Hosp Epidemiol 2010 ;31: 1250-56.

15. Ben-David D, Kordevani R, Keller N, et al. Outcome of carbapenem resistant Klebsiella pneumoniae bloodstream infections. Clin Microbiol Infect. 2012;18:54-60.

16. Tabah A, Koulenti D, Laupland K, et al. Characteristics and determinants of outcome of hospital-acquired bloodstream infections in intensive care units: the EUROBACT International Cohort Study. Intensive Care Med. 2012;38:1930-45.

17. Hussein K, Raz-Pasteur A, Finkelstein R, et al. Impact of carbapenem resistance on the outcome of patients' hospital-acquired bacteraemia caused by Klebsiella pneumoniae. J Hosp Infect. 2013;83: 307-13.

18. Ghafur AK, Vidyalakshmi PR, Kannaian P, et al. Clinical study of carbapenem sensitive and resistant Gram-negative bacteremia in neutropenic and nonneutropenic patients: The first series from India. Indian J Cancer. 2014;51:453-5.

19. Girometti N, Lewis RE, Giannella M, et al. Klebsiella pneumoniae bloodstream infection: epidemiology and impact of inappropriate empirical therapy. Medicine (Baltimore). 2014;93:298-309.

20. Alicino C, Giacobbe DR, Orsi A, et al. Trends in the annual incidence of carbapenem-resistant Klebsiella pneumoniae bloodstream infections: a 8-year retrospective study in a large teaching hospital in northern Italy. BMC Infect Dis. 2015;15:415. 
21. Gomez-Simmonds A, Greenman M, Sullivan SB, et al. Population structure of Klebsiella pneumoniae causing bloodstream infections at a New York City tertiary care hospital: diversification of multidrugresistant isolates. J Clin Microbiol. 2015;53:2060-7.

22. Trecarichi EM, Pagano L, Candoni A, et al. Current epidemiology and antimicrobial resistance data for bacterial bloodstream infections in patients with hematologic malignancies: an Italian multicentre prospective survey. Clin Microbiol Infect. 2015;21: 337-43.

23. Vardakas KZ, Matthaiou DK, Falagas ME, et al. Characteristics, risk factors and outcomes of carbapenem-resistant Klebsiella pneumoniae infections in the intensive care unit. J Infect. 2015;70:592-9.

24. Trecarichi EM, Pagano L, Martino B, et al. Bloodstream infections caused by Klebsiella pneumoniae in onco-hematological patients: clinical impact of carbapenem resistance in a multicentre prospective survey. Am J Hematol. 2016;91:1076-81.

25. Villegas MV, Pallares CJ, Escandon-Vargas K, et al. Characterization and clinical impact of bloodstream infection caused by carbapenemase-producing Enterobacteriaceae in seven Latin American countries. PLoS ONE. 2016;11:e0154092.

26. Veeraraghavan B, Shankar C, Karunasree S, et al. Carbapenem resistant Klebsiella pneumoniae isolated from bloodstream infection: Indian experience. Pathog Glob Health. 2017;111:240-6.

27. Paczosa MK, Mecsas J. Klebsiella pneumoniae: going on the offense with a strong defense. Microbiol Mol Biol Rev. 2016;80:629-61.

28. Iredell J, Brown J, Tagg K. Antibiotic resistance in Enterobacteriaceae: mechanisms and clinical implications. BMJ. 2016;352:h6420.

29. Cassini A, Högberg LD, Plachouras D, et al. Attributable deaths and disability-adjusted lifeyears caused by infections with antibiotic-resistant bacteria in the EU and the European Economic Area in 2015: a population-level modelling analysis. Lancet Infect Dis. 2019;19:56-66.

30. Vardakas KZ, Rafailidis PI, Konstantelias AA, et al. Predictors of mortality in patients with infections due to multi-drug resistant gram-negative bacteria: the study, the patient, the bug or the drug? J Infect. 2013;66:401-14.

31. Viale P, Giannella M, Lewis R, et al. Predictors of mortality in multidrug-resistant Klebsiella pneumoniae bloodstream infections. Expert Rev Anti Infect Ther. 2013;11:1053-63.

32. Schwaber MJ, Klarfeld-Lidji S, Navon-Venezia S, Schwartz D, Leavitt A, Carmeli Y. Predictors of Carbapenem-Resistant Klebsiella pneumoniae Acquisition among Hospitalized Adults and Effect of Acquisition on Mortality. Antimicrob Agents Chemother. 2008; 1028-1033.

33. Tamma PD, Goodman KE, Harris AD, et al. Comparing the outcomes of patients with carbapenemase-producing and non-carbapenemaseproducing carbapenem-resistant enterobacteriaceae bacteremia. Clin Infect Dis. 2017;64:257-64.

34. Tsuji BT, Pogue JM, Zavascki AP, et al. International consensus guidelines for the optimal use of the polymyxins: endorsed by the american college of clinical pharmacy (ACCP), European society of clinical microbiology and infectious diseases (ESCMID), infectious diseases society of America (IDSA), international society for anti-infective pharmacology (ISAP), society of critical care medicine (SCCM), and society of infectious diseases pharmacists (SIDP). Pharmacotherapy. 2019;39:10-39.

35. Giacobbe DR, Saffioti C, Losito AR, et al. Use of colistin in adult patients: a cross-sectional study. J Glob Antimicrob Resist. 2020;20:43-4.

36. Karaiskos I, Lagou S, Pontikis K, Rapti V, Poulakou G. The, "Old" and the "New" Antibiotics for MDR Gram-Negative Pathogens: For Whom, When, and How. Front Public Health. 2019;7:151.

37. Paul M, Leibovici L. Observational studies examining patient management in infectious diseases. Clin Microbiol Infect. 2017;23:127-8.

38. Giacobbe DR, Maraolo AE, Viscoli C. Pitfalls of defining combination therapy for carbapenem-resistant Enterobacteriaceae in observational studies. Eur J Clin Microbiol Infect Dis. 2017;36:1707-9. 